\title{
Aspectos dinámicos en el diseño del anillo de almacenamiento del sincrotrón mexicano
}

\author{
H. J. Villarreal ${ }^{a}$, N. Méndez ${ }^{a}$, J. I. Fuentes ${ }^{a}$, C. E. Mendoza ${ }^{b}$, \\ E. Sánchez ${ }^{c}$, A. Flores-Tlalpa ${ }^{d}$, J. Hernández $^{e}$, M. Moreno $^{f}$, y A. Antillón ${ }^{e}$ \\ ${ }^{a}$ Centro de Investigación en Ciencias, Universidad Autónoma del Estado de Morelos, \\ Av. Universidad 1001, Col. Chamilpa, Cuernavaca, Morelos, 62210, México. \\ ${ }^{b}$ Tecnológico de Zacatepec, Tecnológico 27, \\ Col. Plan de Ayala, 62780, Zacatepec de Hidalgo, Morelos. \\ ${ }^{c}$ Facultad de Ciencias, Universidad Nacional Autónoma de México, \\ México, 04510, Ciudad de México. \\ ${ }^{d}$ Departamento de Bioingeniería y Ciencias, Tecnológico de Monterrey, \\ Puebla, 72453, México. \\ e Instituto de Ciencias Físicas, Universidad Nacional Autónoma de México, \\ Av. Universidad s/n, Col. Chamilpa, Cuernavaca, Morelos, 62210, México. \\ ${ }^{f}$ Instituto de Física, Universidad Nacional Autónoma de México, \\ México, 04510, Ciudad de México.
}

Received 16 October 2021; accepted 24 November 2021

Se investigan varios diseños de redes magnéticas para la fuente de luz sincrotrón mexicana, los cuales hacen uso de algunos recursos de diseño de las actualizaciones recientes de fuentes de luz sincrotrón, tales como celdas acromáticas múltiples, dipolos con gradiente longitudinal, dipolos invertidos y esquemas híbridos. Mediante el uso de bloques fijos de imanes (tipo LEGO), también, se investiga la posibilidad de reducir aún más la emitancia de algunos diseños.

Descriptores: Redes magnéticas; luz sincrotrón.

Various magnetic lattice designs for the Mexican synchrotron light source are investigated, which use some design resources from recent synchrotron light source upgrades, such as multiple achromatic cells, longitudinal gradient dipoles, inverted dipoles, and hybrid schemes. By using fixed blocks of magnets (LEGO type), the possibility of further reducing the emittance of some designs is also being investigated.

Keywords: Magnetic lattice designs; synchrotron light source.

DOI: https://doi.org/10.31349/SuplRevMexFis.3.010603

\section{Introducción}

La idea de contar en México con una fuente de luz sincrotrón es un proyecto que ha estado presente desde hace más de diez años, en varias etapas de elaboración. La formulación del proyecto se ha ido consolidando desde diversas aristas con una participación internacional muy importante. Personal de algunos laboratorios como ESRF, SLS, ALBA, DIAMOND, NSLS-II, APS, ALS y SLAC han sido fuente de inspiración y apoyo al proyecto, a la vez que ha sido posible tomar elementos esenciales de sus respectivos proyectos de actualización para plasmarlos en modelos del sincrotrón mexicano para que sean viables. Para simplificar la lectura, en la Tabla I se dan los significados, acrónimos y nombres contenidos en este artículo.

La dirección marcada por el esquema de celdas acromáticas múltiples (MBA), y su uso exitoso en MAX IV, ha mostrado ser adecuada para transitar a fuentes de luz limitadas por difracción (DLSR) que proporcionan dos o tres órdenes de magnitud más brillantez y mayor coherencia que las fuentes de $3 .^{a}$ generación, aunque su uso no está exento de producir una serie de fenómenos no deseados que deben controlarse. Las celdas MBA requieren imanes cuadrupolares muy intensos que producen aberraciones cromáticas que son difíci- les de corregir. Se requiere también de imanes sextupolares intensos para su correción cromática, lo cual ocasiona, entre otras dificultades, mayores efectos de dinámica no lineal que en general disminuyen la apertura dinámica con posibles consecuencias en problemas de inyección. Por esto se requiere utilizar programas de simulación con cálculos de alta precisión y métodos sofisticados de optimización. Para conseguir campos más intensos se reducen las aperturas transversales de los imanes, lo que conlleva retos en su diseño así como en el diseño del sistema de vacío [1].

En este trabajo se exploran modelos que además de contener celdas MBA incorporan otros conceptos de gran relevancia en el diseño como dipolos invertidos (RB), usado extensivamente en SLS-2, dipolos con gradiente de campo longitudinal (LGB), en ESRF-ESB, SLS-2 y desarrollos posteriores de actualizaciones de sincrotrones (APS-II, Diamond-II, SOLEIL-II, PETRA IV) y de nuevos proyectos (HEPS). Se agrega a lo anterior otro ingrediente modular (tipo LEGO) que permite adicionar o suprimir bloques de imanes del interior de la celda, con lo cual se puede explorar el espacio mostrado en la Fig. 15 de una forma sistemática.

Los modelos que aquí se exhiben representan la versión simple en su diseño de dinámica lineal y no lineal. Se requiere de estudios más elaborados en varios rubros incluyendo 
TABLA I. Acrónimos y abreviaturas.

\begin{tabular}{|c|c|}
\hline & Significado \\
\hline ESRF-EBS & European Synchrotron Radiation \\
\hline & Facility-Extremely Brilliant Source \\
\hline SLS & Swiss Light Source \\
\hline ALBA & Sincrotrón español \\
\hline DIAMOND & Sincrotrón del Reino Unido \\
\hline NSLS-II & $\begin{array}{l}\text { National Synchrotron Light Source II } \\
\text { (Brookhaven) }\end{array}$ \\
\hline APS & Advanced Photon Source (Argonne) \\
\hline ALS & Advanced Light Source (Berkeley) \\
\hline SLAC & Stanford Linear Accelerator Center \\
\hline MAX IV & Sincrotrón Sueco \\
\hline SOLEIL & Sincrotrón francés \\
\hline PETRA IV & Sincrotrón alemán \\
\hline HEPS & High Energy Photon Source (China) \\
\hline CLS & Canadian Light Source \\
\hline MLS & Mexican Light Source \\
\hline MBA & Multi-Bend Achromat \\
\hline LGB & Longitudinal Gradient Bends \\
\hline LGBwAB & Longitudinal Gradient Bends with antibends \\
\hline $\mathrm{RB}(\mathrm{AB})$ & Reverse Bends (Anti-Bends) \\
\hline SB & Super Bends \\
\hline DLSR & Diffraction Limited Storage Rings \\
\hline OPA & Lattice design code \\
\hline AA & Anillo de almacenamiento \\
\hline LEGO & Usado como similitud de modular \\
\hline
\end{tabular}

dinámica no lineal, vida media del haz, tolerancias y efectos colectivos para determinar cuál de ellos es buena opción para el sincrotrón mexicano desde el punto de vista dinámico, así como su compatibilidad con opciones de sistemas de inyección como sincrotrón acelerador (booster) o acelerador lineal (linac).

\section{Estado actual de los sincrotrones de $3 .^{a}$ ge- neración y sus actualizaciones}

En esta sección se pretende dar una idea del estado del arte en que se encuentra el diseño de las últimas fuentes de luz sincrotrón. Para ello haremos uso de la figura de la página 23 presentada en la Ref. [1] conocida también como Bartolini chart. A lo largo de la línea azul se encuentran agrupados sincrotrones que se catalogan como de $3 .^{a}$ generación. La línea roja sólida se establece sobre una zona de sincrotrones que utilizan sobre todo el esquema de MultiBend Achromat (MBA) introducido en las referencias [2-4]. En esta dirección se mueven los nuevos diseños de fuentes de luz con haces de electrones de muy baja emitancia programados para proveer

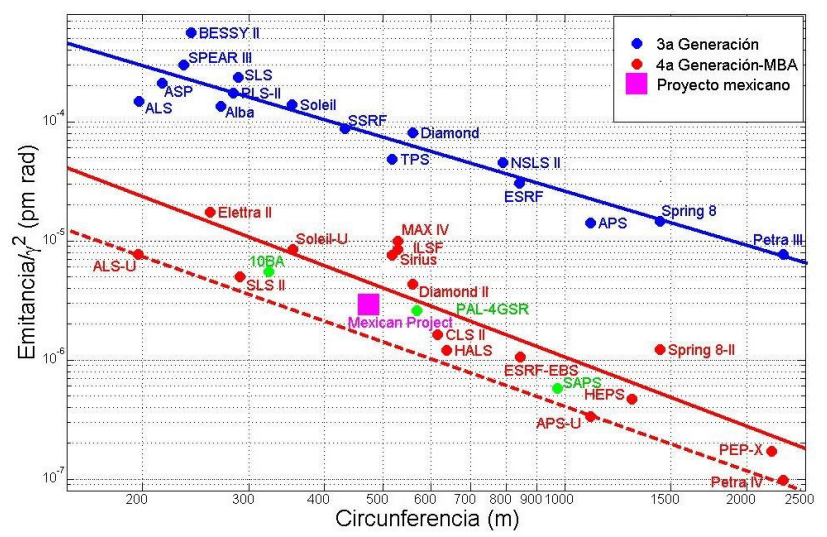

FIGURA 1. Se muestran varios proyectos de fuentes de luz agrupados en tres regiones principales. La línea azul relacionada con sincrotrones de $3 .^{a}$ generación, la línea roja sólida agrupa a la $4 .^{a}$ generación y cerca de la línea roja discontinua están los proyectos que incorporan ideas novedosas de diseño y nuevas tecnologías.

a los usuarios de haces de luz de alta brillantez y coherencia. Sobre la línea roja discontinua se aglutinan los proyectos cuyo diseño es más demandante de los progresos tecnológicos. Sólo uno de ellos, ESRF-EBS, se encuentra ya en funcionamiento. Hay un cuadro magenta que señala la zona de parámetros en la cual creemos que es factible posicionar el sincrotrón mexicano. A lo largo de este trabajo mostraremos diseños realizados en los últimos años que muestran parcialmente que esta selección, circunferencia-emitancia, es técnicamente posible.

\section{Explorando modelos para la fuente de luz mexicana (MLS)}

Las ideas principales consideradas para los diseños aquí propuestos provienen sobre todo de proyectos a lo largo de la línea roja discontinua, que son los proyectos de actualización de ALS y SLS así como ESRF-EBS que ya está en funcionamiento.

\subsection{Modelo tipo ALS-II}

Durante el III Mexican Workshop on Accelerator Physics realizado en Huatulco, expertos participantes [5] sugirieron $3 \mathrm{Gev}, 300 \mathrm{~m}$ y $300 \mathrm{pm} \cdot \mathrm{rad}$ como parámetros factibles para el diseño del sincrotrón mexicano. El diseño 9BA que ALS consideraba para su actualización [6] resultó atractivo para el objetivo mencionado. En la Fig. 2 se muestra la distribución magnética encontrada (en azul se indican los dipolos y en rojo los cuadrupolos) con sus funciones $\beta_{x}$ (azul), $\beta_{y}$ (rojo), y su función de dispersión $\eta$ (verde) correspondientes. En la Tabla II se muestran algunos parámetros relevantes del modelo.

Se procuró mantener las intensidades máximas de los cuadrupolos en valores similares a los usados en ALS-II. Estudios posteriores sobre la dinámica no lineal llevados a cabo con OPA [7] muestran que la apertura dinámica se reduce a 


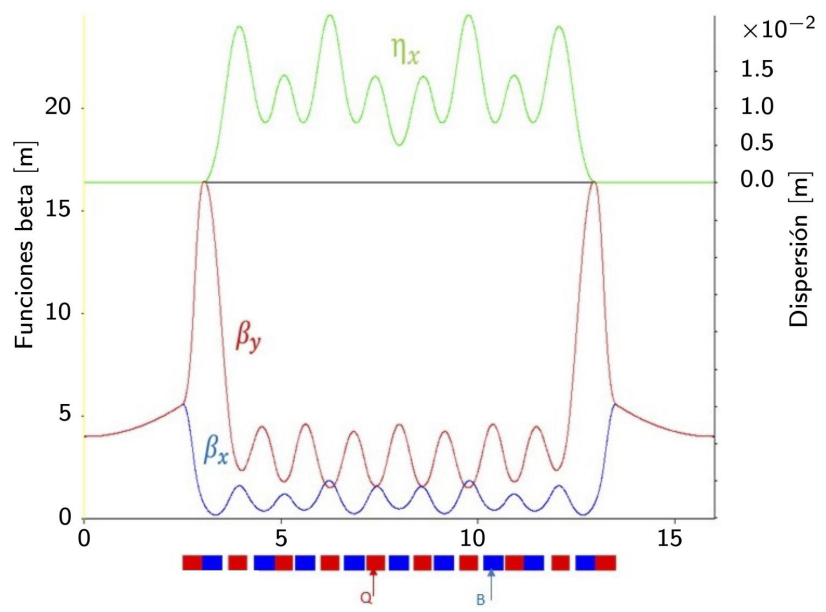

FIGURA 2. Funciones ópticas obtenidas al adaptar al sincrotrón mexicano, el modelo ALS-II con celdas 9BA. En la parte inferior se muestra la distribución de dipolos (azul) y cuadrupolos (rojo). En la Tabla II se muestran los parametros relevantes.

TABLA II. Parámetros principales del modelo 9BA de ALS-II considerado en la Fig. 2.

\begin{tabular}{cc}
\hline Parámetro & Valor \\
\hline Energía & $3 \mathrm{GeV}$ \\
Circunferencia & $320 \mathrm{~m}$ \\
Número de celdas & 20 \\
Tono betatrón $\left(\nu_{x}, \nu_{y}\right)$ & $57.56,14.54$ \\
Cromaticidad natural $\left(\xi_{x}, \xi_{y}\right)$ & $-72,-90$ \\
Emitancia $\left(\epsilon_{x}\right)$ & $84 \mathrm{pm} \cdot \mathrm{rad}$ \\
Factor de compactación de momento $\left(\alpha_{c}\right)$ & $1.5 \times 10^{-4}$ \\
\hline
\end{tabular}

dimensiones menores de $1 \mathrm{~mm}$, comprometiendo entre otros factores, al proceso de inyección. Para resolver el problema de inyección en su diseño de baja emitancia, ALS-II contempla un anillo de acumulación del cual se intercambian paquetes de electrones (on-axis swap-out injection) con el anillo de almacenamiento (AA).

\subsection{Modelos tipo ESRF-EBS}

El diseño híbrido de ESRF-EBS [8,9] parece ser sencillo y atractivo para usarse en otros proyectos de sincrotrones [10-12]. La prominencia en la función de dispersión es atractiva para la correción cromática si adicionalmente se usa la transformación $-I$ para sextupolos colocados en estas regiones. Esto hace que este modelo sea explorado en múltiples variantes como alternativa para el sincrotrón mexicano. A continuación se describen brevemente cuatro de estas alternativas.

\subsubsection{Modelo tipo ESRF-EBS de 16 celdas, $422 \mathrm{~m}$}

La primera versión explorada se obtiene al reducir la circunferencia de ESRF-EBS a la mitad, a la vez que se mantiene su estructura magnética. En la Fig. 3 se muestra, en la parte

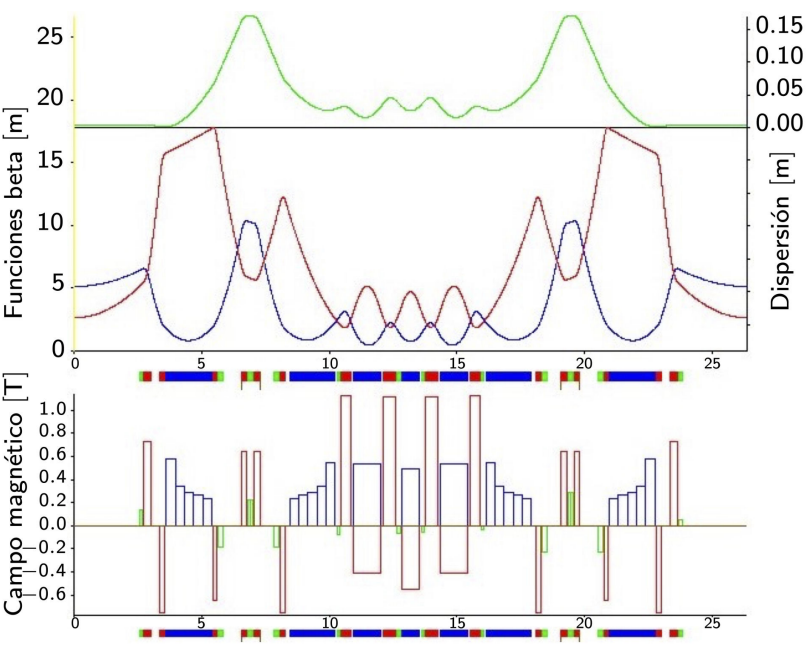

FIGURA 3. (Bloques superiore) Estructura magnética y funciones $\beta$ у $\eta$ correspondientes. (Bloques inferiores) Intensidad... (en Fig. 3) de los campos de los elementos magnéticos. La circunferencia mide $422 \mathrm{~m}$ con 16 celdas. En la Tabla III se dan más detalles.

TABLA III. Parámetros principales del modelo tipo ESRF-EBS considerado en la Fig. 3.

\begin{tabular}{cc}
\hline Parámetro & Valor \\
\hline Energía & $3 \mathrm{GeV}$ \\
Circunferencia & $422 \mathrm{~m}$ \\
Número de celdas & 16 \\
Tono betatrón $\left(\nu_{x}, \nu_{y}\right)$ & $37.78,13.83$ \\
Cromaticidad natural $\left(\xi_{x}, \xi_{y}\right)$ & $-50.3,-40.7$ \\
Emitancia $\left(\epsilon_{x}\right)$ & $274 \mathrm{pm} \cdot \mathrm{rad}$ \\
Factor de compactación de momento $\left(\alpha_{c}\right)$ & $3.7 \times 10^{-4}$ \\
\hline
\end{tabular}

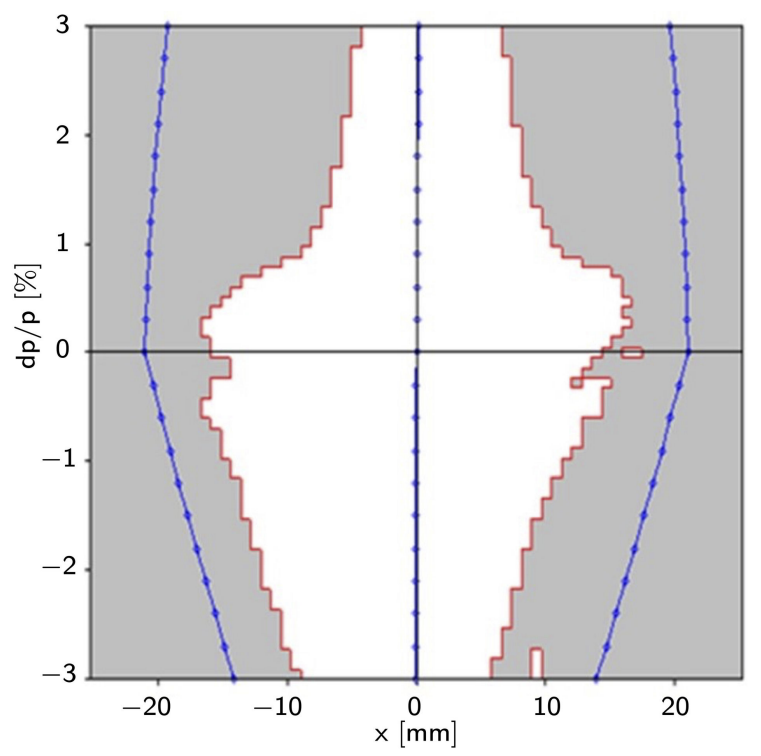

Figura 4. Apertura de momento al inicio de la celda para el modelo tipo ESRF-EBS de la Fig. 3. 
superior el arreglo magnético y las funciones ópticas correspondientes, y en la parte inferior la intensidad de los campos de los elementos magnéticos. Siguiendo el mismo código de colores de la Fig. 2 para dipolos y cuadrupolos, mientras que los sextupolos se representan en verde.

\subsubsection{Modelo tipo ESRF-EBS de 16 celdas con SB, $422 \mathrm{~m}$}

En el modelo de la Fig. 3 se tienen 16 secciones rectas. Si una se usa para la inyección y otra para la cavidad de radiofrecuencia, se termina con 14 espacios para líneas experimentales provenientes de dispositivos de inserción. Una forma de aumentar el número de líneas que provean de rayos $\mathrm{X}$ duros es colocando SB. En el modelo de esta sección, mostrado en

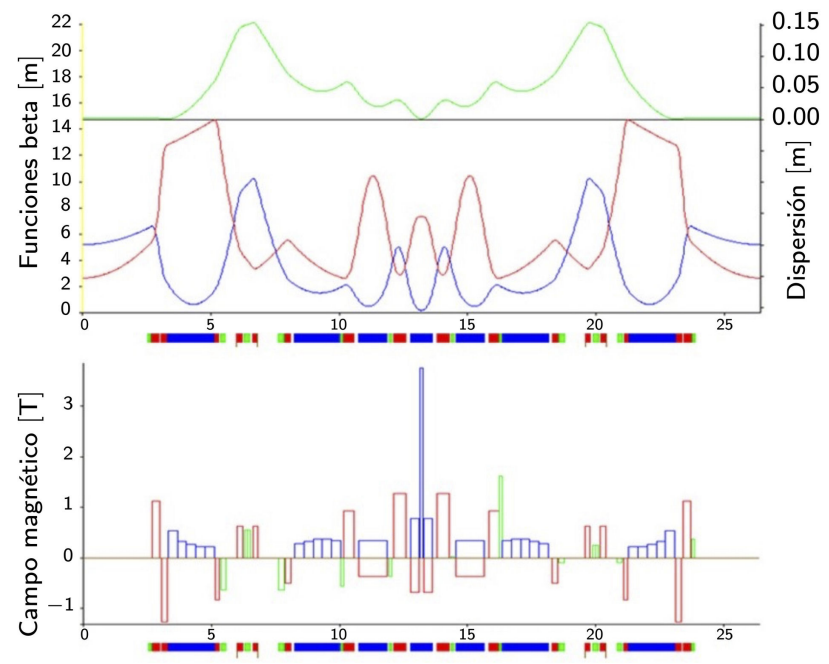

FIGURA 5. (Bloque superior) Estructura magnética y funciones $\beta$ y $\eta$ correspondientes. (Bloque inferior) Intensidad... (en Fig. 5) de los campos de los elementos magnéticos. La longitud de la circunferencia es $422 \mathrm{~m}$ con 16 celdas. Al centro de la celda hay un SB de $\sim 4 T$. En la Tabla IV se muestran más detalles.

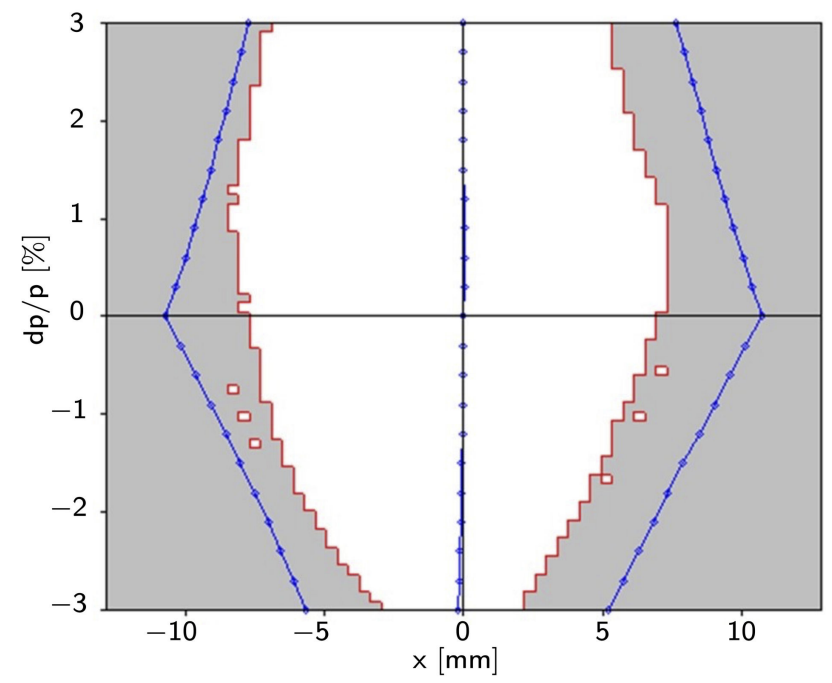

Figura 6. Apertura de momento al inicio de la celda para el modelo tipo ESRF-EBS de la Fig. 5.
TABLA IV. Parámetros principales del modelo considerado en la Fig. 5.

\begin{tabular}{cc}
\hline Parámetro & Valor \\
\hline Energía & $3 \mathrm{GeV}$ \\
Circunferencia & $422 \mathrm{~m}$ \\
Número de celdas & 16 \\
Tono betatrón $\left(\nu_{x}, \nu_{y}\right)$ & $39.31,14.07$ \\
Cromaticidad natural $\left(\xi_{x}, \xi_{y}\right)$ & $-61.8,-45.5$ \\
Emitancia $\left(\epsilon_{x}\right)$ & $223 \mathrm{pm} \cdot \mathrm{rad}$ \\
Factor de compactación de momento $\left(\alpha_{c}\right)$ & $3.8 \times 10^{-4}$ \\
\hline
\end{tabular}

TABLA V. Parámetros principales del modelo considerado en la Fig. 7.

\begin{tabular}{cc}
\hline Parámetro & Valor \\
\hline Energía & $3 \mathrm{GeV}$ \\
Circunferencia & $392 \mathrm{~m}$ \\
Número de celdas & 16 \\
Tono betatrón $\left(\nu_{x}, \nu_{y}\right)$ & $39.39,17.37$ \\
Cromaticidad natural $\left(\xi_{x}, \xi_{y}\right)$ & $-42.1,-46.51$ \\
Emitancia $\left(\epsilon_{x}\right)$ & $226 \mathrm{pm} \cdot \mathrm{rad}$ \\
Factor de compactación de momento $\left(\alpha_{c}\right)$ & $3.2 \times 10^{-4}$ \\
\hline
\end{tabular}

la Fig. 5, se propone colocar un superbend por celda con lo cual se tiene en total 30 espacios para líneas experimentales. La Fig. 5 muestra tres bloques, los dos superiores con las funciones ópticas del problema lineal y la inferior con las intensidades de los elementos magnéticos involucrados. El dipolo central se dividió en tres, con el SB de $\sim 4 T$ en la parte central.

Un estudio preliminar de la apertura en momento al inicio de la sección recta, que también da idea de la apertura dinámica, se llevó a cabo con el programa OPA y el resultado se muestra en la Fig. 6.

\subsubsection{Modelo tipo ESRF-EBS de 16 celdas 9BA, $392 \mathrm{~m}$}

Los dos modelos anteriores son del tipo 7BA como el diseño de ESRF-EBS. Los dos siguientes modelos que se estudian son del tipo 9BA. Aparte de ALS-II se tiene un diseño para CLS-II [13] en esta categoría. Manteniendo la estructura general de ESRF-EBS, para el primer modelo 9BA se adiciona un par de celdas internas para lograr el esquema 9BA que proporciona una emitancia menor que el caso en (3.2.2). La estructura magnética y sus funciones lineales se muestran en la Fig. 7 y los parámetros básicos de este modelo 9BA se muestran en la Tabla V. Por otro lado, los estudios preliminares con OPA de la apertura local de momento cuando se incorporan los sextupolos se muestran en la Fig. 8. Las fases usadas $\Delta \phi_{x}, \Delta \phi_{y}$ por celda parecen atractivas para mejorarse con programas tales como Elegant [14]. 


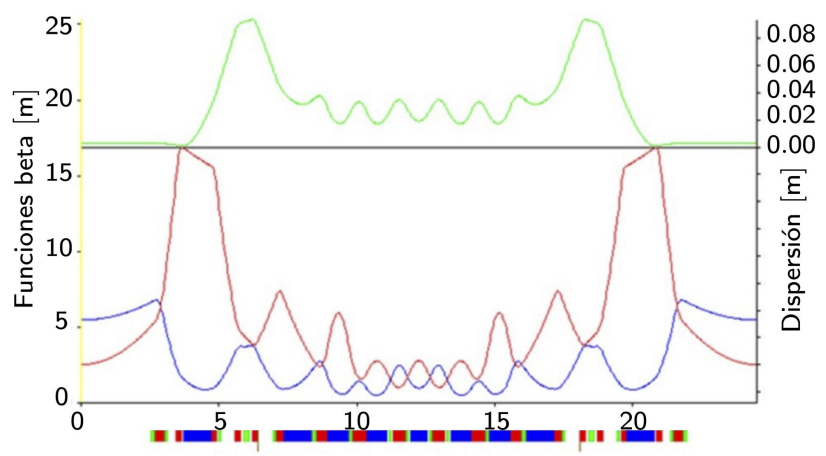

FIGURA 7. Estructura magnética y las funciones $\beta$ y $\eta$ correspondientes para la celda 9BA. La longitud de la circunferencia es 392 $\mathrm{m}$ con 16 celdas. Las Tabla V contiene mayor detalle.

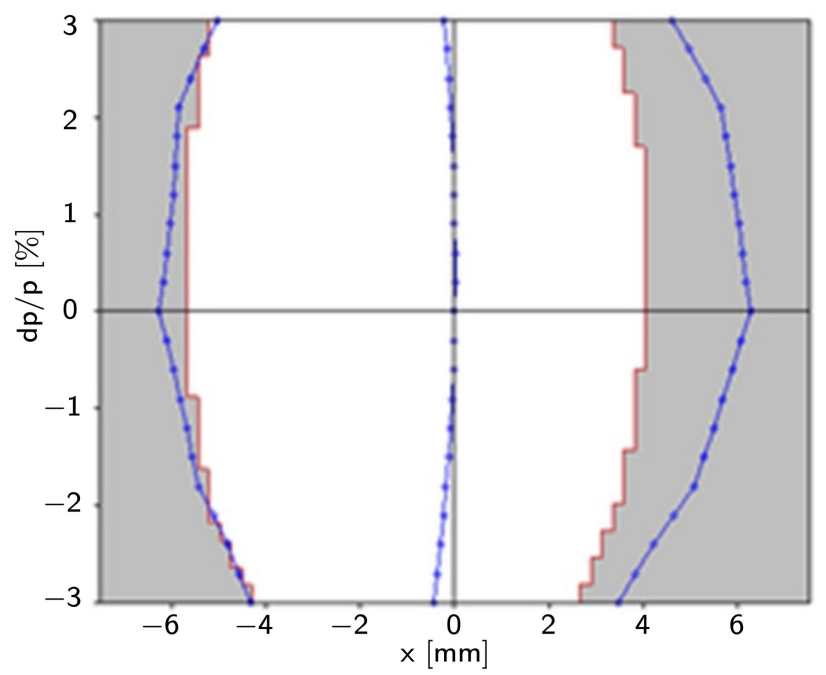

Figura 8. Apertura de momento al inicio de la celda para el modelo tipo ESRF-EBS de 9BA de la Fig. 7.

\subsubsection{Modelo tipo ESRF-EBS de 16 celdas $9 B A+A B, 426$ $m$}

El último modelo tipo ESRF que se presenta es como el de la Fig. 7 al cual se le adicionan AB, logrando una emitancia por abajo de los $100 \mathrm{pm} \cdot$ rad. Este modelo se muestra en la Fig. 9. Las dos gráficas superiores detallan la estructura magnética y las funciones ópticas de diseño y la inferior muestra la intensidad de los campos magnéticos involucrados en cada elemento de la celda. Aunque la distorsión del espacio fase horizontal parece variar suavemente con la amplitud a $\Delta p / p=0$, existe una cadena de resonancias de orden alto que limitan la region de estabilidad de la inestable o posiblemente caótica. Este fenómeno se puede apreciar en la Fig. 10.

En la categoría de este tipo de celda, hay otros trabajos recientes [15] que muestran modelos 9BA con y sin $\mathrm{AB}$, con emitancia por debajo de $100 \mathrm{pm} \cdot \mathrm{rad}$, que desde el punto de vista de la apertura dinámica parecen ser robustos a cambio de incrementar el tamaño de la celda y por tanto de la circunferencia del AA.

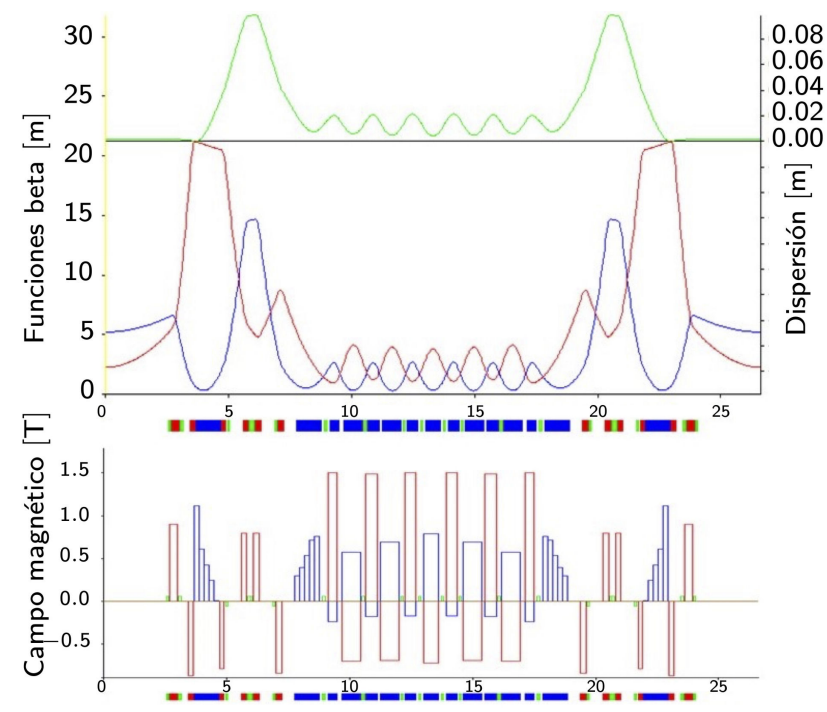

FIgURA 9. Diseño tipo ESRF de 9BA con AB incluidos. En los dos bloques superiores se muestra la estructura magnética y las funciones $\beta$ y $\eta$ correspondientes. En el bloque inferior se muentra la intensidad de los campos de los elementos magnéticos. La longitud de la circunferencia es $426 \mathrm{~m}$ con 16 celdas. La Tabla VI contiene mayor detalle.

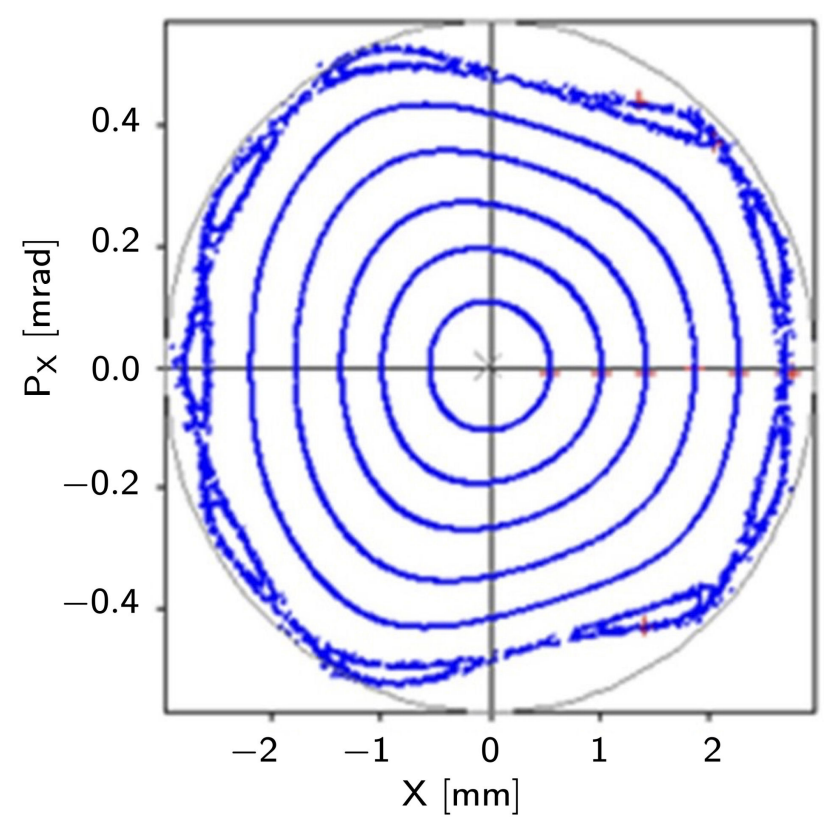

FIGURA 10. Espacio fase horizontal para $\Delta p / p=0$ del modelo de la Fig. 9 que contiene elementos no lineales. La distorsión de las órbitas parece variar suavemente con la amplitud, sin embargo, existe una cadena de resonancias de orden alto que limitan la región de estabilidad.

\subsection{Modelo tipo SLS-2}

Una opción que ha surgido en el análisis de los diferentes modelos es el usar en la medida de lo posible, los avances tecnológicos que se han realizado para satisfacer los requerimientos de SLS-2 (7BA, $288 \mathrm{~m}, 2.4 \mathrm{GeV}, \sim 130 \mathrm{pm} \cdot \mathrm{rad}$ ). 
TABLA VI. Parámetros principales del modelo considerado en la Fig. 9.

\begin{tabular}{cc}
\hline Parámetro & Valor \\
\hline Energía & $3 \mathrm{GeV}$ \\
Circunferencia & $426 \mathrm{~m}$ \\
Número de celdas & 16 \\
Tono betatrón $\left(\nu_{x}, \nu_{y}\right)$ & $54.91,21.28$ \\
Cromaticidad natural $\left(\xi_{x}, \xi_{y}\right)$ & $-95.0,-64.21$ \\
Emitancia $\left(\epsilon_{x}\right)$ & $73 \mathrm{pm} \cdot \mathrm{rad}$ \\
Factor de compactación de momento $\left(\alpha_{c}\right)$ & $8.8 \times 10^{-5}$ \\
\hline
\end{tabular}

TABLA VII. El uso en dos diseños. La celda optimizada de la Fig. 11, cuando se hace el cambio de energía de $2.4 \mathrm{GeV}$ a $3 \mathrm{GeV}$ con la idea de conservar la tecnología de los imanes de SLS-2, conduce al siguiente cuadro comparativo de parámetros principales de los dos sincrotrones. El término referencia(SLS-2) se refiere al diseño modificado de SLS-2 a $2.4 \mathrm{GeV}$.

\begin{tabular}{ccc}
\hline Parámetro & referencia(SLS-2) & MLS \\
\hline Energía & $2.4 \mathrm{GeV}$ & $3 \mathrm{GeV}$ \\
Circunferencia & $292 \mathrm{~m}$ & $365 \mathrm{~m}$ \\
Número de celdas & 12 & 15 \\
Tono betatrón $\left(\nu_{x}, \nu_{y}\right)$ & $40.80,16.24$ & $50.98,20.21$ \\
Cromaticidad natural $\left(\xi_{x}, \xi_{y}\right)$ & $-128.22,-34.42$ & $-161,-43$ \\
Emitancia $\left(\epsilon_{x}\right)$ & $54 \mathrm{pm} \mathrm{rad}$ & $43 \mathrm{pm} \mathrm{rad}$ \\
Factor de compactación & & \\
de momento $\left(\alpha_{c}\right)$ & $9.6 \times 10^{-5}$ & $6.1 \times 10^{-5}$ \\
\hline
\end{tabular}

Con ello se puede reducir la duración de desarrollos similares logrando un Reporte de Diseño Conceptual en menos tiempo. Considerando esta idea, se estudia la posibilidad de usar la misma tecnología de imanes de SLS-2 en un sincrotrón de mayores dimensiones y energía que SLS-2.

\subsubsection{Celda interna de SLS-2 con AB en LGB (LGBwAB)}

El estudio comprende la posibilidad de reducir la emitancia de SLS-2 (de $\sim 130 \mathrm{pm} \cdot \mathrm{rad}$ ), lo cual se logra a $\sim 54 \mathrm{pm}$. rad mediante la introducción de un $\mathrm{AB}$ en cada dipolo LGB (LGBwAB), como se describe teóricamente en la sección inferior de la Fig. 11. La gráfica superior muestra la celda interna de SLS-2, con una optimización adicional en la emitancia, que proporciona las funciones $\beta$ y $\eta$ mostradas. Usualmente los SB sirven para tener líneas de luz de rayos X duros. El tener $5 \mathrm{SB}$ por celda, como se usa en el modelo, puede resultar en un conglomerado de SB de donde sea difícil extraer luz sincrotrón y por tanto el número debería reducirse en un modelo más práctico.

Más aún, dado que la optimización se hizo considerando únicamente la emitancia como función objetivo, sin otra limitación, no es extraño que la respuesta sea un LGwAB con campos magnéticos grandes, aunque alcanzables con tecnología superconductora. El punto es, si se puede realizar un

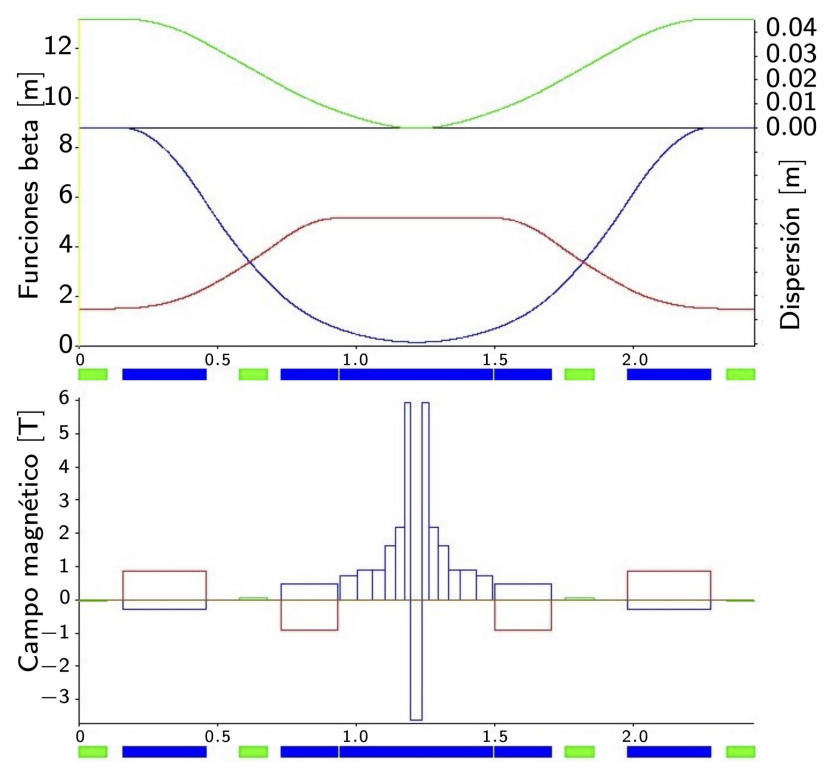

FIgURA 11. Celda interna de SLS-2 optimizada con el requerimiento de menor emitancia. El algoritmo introduce un $\mathrm{AB}$ en el LGB, como lo indica la figura inferior. Esto posibilita la reducción de emitancia de $\sim 130 \mathrm{pm} \cdot \operatorname{rad}$ en SLS-2 a $\sim 54 \mathrm{pm} \cdot \operatorname{rad}$ a 2.4 $\mathrm{GeV}$. La gráfica superior muestra las funciones $\beta$ y $\eta$ para la celda interna optimizada.

diseño técnico apropiado. Consideramos que se puede relajar el diseño abriendo espacio entre los dipolos de campo extremo y/o reduciendo la intensidad de ellos para llevarlos, por ejemplo, a la categoría de imanes normales, hasta que sea técnicamente factible su construcción. El precio que se paga en este proceso es el aumento de la función objetivo. Por lo anterior, consideramos que lo importante para el estudio de esta sección es mantener un esquema fijo y utilizarlo en la forma propuesta, y ver la tendencia general de los resultados, independientemente de la factibilidad técnica de construir un LGwAB como el considerado aquí.

Una vez que se logra lo expuesto en el punto inicial, se incrementa la energía a $3 \mathrm{GeV}$ para lo cual es necesario aumentar la circunferencia dejando los campos dipolares iguales. En la Tabla VII se hace el cuadro comparativo de ambas configuraciones, $2.4 \mathrm{GeV}, 290 \mathrm{~m}$ y $3 \mathrm{GeV}, 365 \mathrm{~m}$.

\subsubsection{Estructura de la celda en bloques tipo LEGO}

Lo que se tiene hasta este punto en relación al modelo SLS-2 y con el desarrollo de la Fig. 11, es un esquema de bloques ensamblados que conforman SLS-2. En lenguaje figurado se puede pensar en un esquema modular de piezas tipo LEGO que quitamos y ponemos para establecer estructuras. En este sentido, si quitamos bloques internos de la celda se puede generar una variedad de esquemas de los cuales sólo se mencionan los mostrados en la Fig. 12.

Esta figura muestra tres estructuras de modelos, en la superior sólo se dejan tres de las cinco celdas internas asociadas a SLS-2, del tipo de la Fig. 11. El módulo central sólo tiene una celda interna y el inferior no tiene celdas internas. En la 


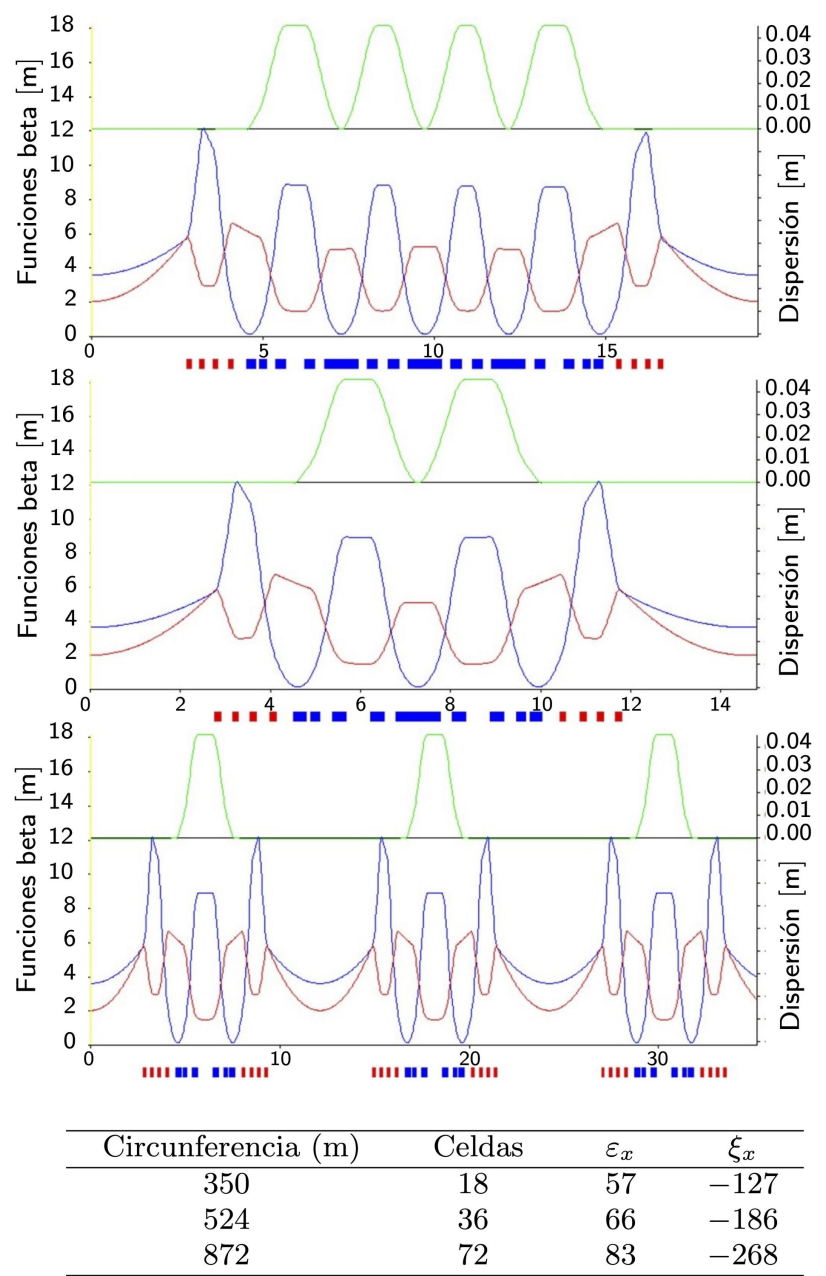

FIGURA 12. Se muestran tres modelos con 3,1 y 0 celdas internas tipo Fig. 11, de las 5 que usualmente tiene el 7BA de SLS-2 (a $290 \mathrm{~m}$ ). La tabla inferior en la figura muestra la circunferencia, el número de celdas que constituyen el anillo del sincrotrón, la emitancia horizontal y la cromaticidad horizontal. El modelo inferior tiene alrededor del doble de la cromaticidad que el modelo superior, indicando mayores problemas no lineales. Las circunferencias correspondientes son, de arriba a abajo, 350, 524 y $872 \mathrm{~m}$. Los demás parámetros se relacionan con las figuras en el mismo orden.

tabla inferior de la figura se reporta la circunferencia de cada sincrotrón, el número de celdas que contiene, su emitancia y su cromaticidad horizontal. El escalamiento de la emitancia al aumentar el número de celdas, disminuyendo con ello la intensidad del campo de los dipolos, va como circunferencia ${ }^{-3}$. Este no es el escalamiento que vemos en la tabla de la Fig. 11. Las tres subgráficas de esta figura se obtienen con el campo fijo de los dipolos participantes. Por ejemplo, en la subgráfica inferior el ángulo de desviación por cada una de las 72 celdas es $5^{\circ}$. Es decir, cada extremo desvía $2.5^{\circ}$ en las tres subgráficas y cada celda interna desvía $5^{\circ}$ en las dos subgráficas restantes. El último parámetro, la cromaticidad, es el que tiene un cambio más significativo y por tanto, de arriba a abajo, se espera que la complejidad de la dinámica aumente, siendo la del modelo inferior la más complicada.
TABLA VIII. Los parámetros que se muestran en esta tabla corresponden a los dos modelos que surgen de la propuesta [17]. El modelo 1 se muestra en la Fig. 13 y el modelo 2 en la Fig. 14.

\begin{tabular}{ccc}
\hline Parámetro & Modelo 1 & Modelo 2 \\
\hline Energía & $3 \mathrm{GeV}$ & $3 \mathrm{GeV}$ \\
Circunferencia & $360 \mathrm{~m}$ & $405 \mathrm{~m}$ \\
Número de celdas & 16 & 18 \\
$\mathrm{SB}$ & $3.6 \mathrm{~T}$ & $3.2 \mathrm{~T}$ \\
Tono betatrón $\left(\nu_{x}, \nu_{y}\right)$ & $52.28,20.25$ & $58.82,22.74$ \\
Cromaticidad natural $\left(\xi_{x}, \xi_{y}\right)$ & $-133,-39$ & $-150,-44$ \\
Emitancia $\left(\epsilon_{x}\right)$ & $92 \mathrm{pm} \cdot \mathrm{rad}$ & $65 \mathrm{pm} \cdot \mathrm{rad}$ \\
Factor de compactación & & \\
de momento $\left(\alpha_{c}\right)$ & $5.3 \times 10^{-5}$ & $4.2 \times 10^{-5}$ \\
\hline
\end{tabular}

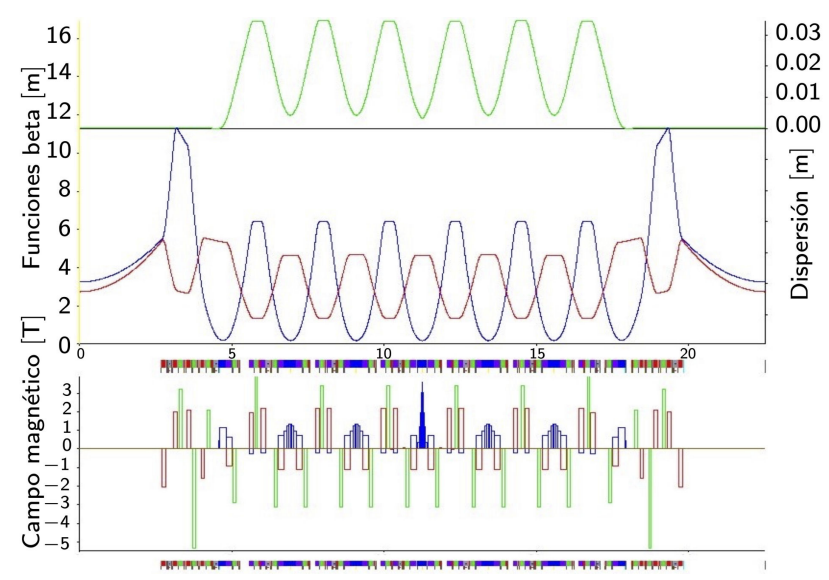

FIGURA 13. Modelo 1 formado con 16 celdas de la versión v.b025 [16] de SLS-2, como fue sugerido por A. Wrulich [17]. Tiene capacidad para 30 líneas experimentales, 14 de dispositivos de inserción y 16 de SB de 3.6 T.

\subsubsection{Modelos extendidos de SLS-2}

En este trabajo también se aborda la siguiente posibilidad de diseño preliminar para el sincrotrón mexicano, usando información amablemente compartida por A. Streun [16]. Las dos versiones que se presentan fueron establecidas por A. Wrulich del PSI [17], y por completez se detallan en esta sección. La primera se muestra en la Fig. 13 donde el anillo principal se constituye de 16 celdas que dan $360 \mathrm{~m}$ de circunferencia con $92 \mathrm{pm}$.rad de emitancia. La celda incluye un SB de 3.6 $\mathrm{T}$ con lo cual se tiene la posibilidad de establecer 30 líneas experimentales, 14 de dispositivos de inserción y 16 SB.

Hacer cuentas de disponibilidad de líneas experimentales de la forma como se ha hecho, es muy optimista porque es posible que el sistema de radiofrecuencia requiera espacio adicional para su operación o para respaldo (ver Fig. 2.60 de la Ref. [18]). Además, se deben acomodar los otros sistemas necesarios para la operación del sincrotrón, como sistema de 


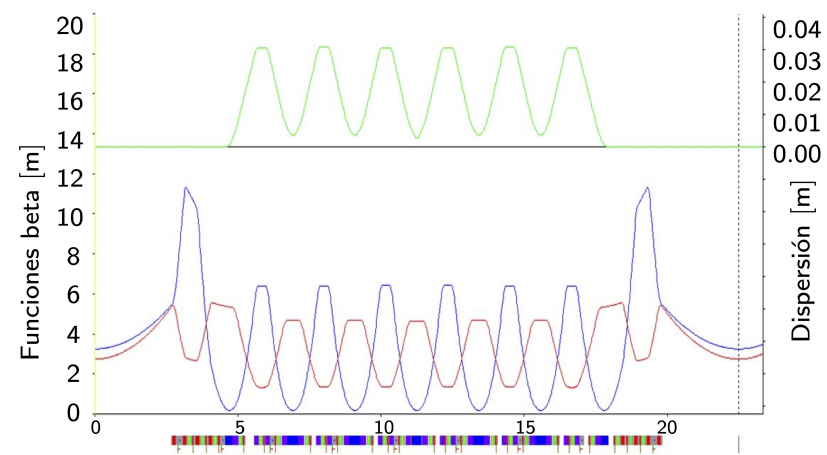

FIGURA 14. Modelo 2 formado con 18 celdas de la versión v.b025 [16] de SLS-2, como fue sugerido por A. Wrulich [17]. Tiene capacidad para 34 líneas experimentales, 16 de dispositivos de inserción y 18 de SB de $3.2 \mathrm{~T}$.

vacío (bombas y medición) y diagnóstico de haz, que por tener dimensiones menores pueden distribuirse en las celdas y probablemente no requieran un espacio mayor especial (versión v.b025 mencionada en la Ref. [19]).

El segundo modelo tiene 18 celdas y se muestra en la Fig. 14. En $405 \mathrm{~m}$ se logra una emitancia de $65 \mathrm{pm}$ y tiene una capacidad de 34 líneas experimentales. Aún no se tiene una optimización no lineal apropiada, pero la que se hereda de SLS-2, conservando el mismo esquema y valores de sextupolos, muestra una posible apertura dinámica del orden de $2 \mathrm{~mm}$. Mayor detalle de algunos parámetros relevantes de ambos modelos se muestran en la Tabla VIII.

\subsection{Modelo tipo ESRF-EBS + SLS-2}

Para este trabajo se han seguido con atención los últimos desarrollos en el diseño de celdas de fuentes de luz sincrotrón tales como MBA [18], LGB [19] y RB [20] que pueden producir una reducción de la emitancia. Varios proyectos hacen uso eficiente de estos conceptos de diseño, ESRF-EBS, SLS2, APS-II y HEPS y también de la relativa simplicidad del esquema híbrido de ESRF-EBS que contiene elementos no lineales en las prominencias de dispersión, lo cual facilita la correción cromática con menores intensidades de sextupolos y por tanto menores efectos no lineales, en beneficio de una mayor apertura dinámica. Entre los sincrotrones que han incluido un diseño híbrido con elementos adicionales se encuentran Diamond-II, APS-II, HEPS, SOLEIL-II, PETRA IV y ALS-II, lo cual refuerza nuestro interés en un planteamiento de celda para el sincrotrón mexicano, que incluya una celda MBA híbrida, con LGB y RB.

En esta sección convergen ideas usadas en secciones anteriores principalmente entre los modelos de ESRF-EBS y SLS-2, que pueden empatar con lo discutido en el párrafo anterior. La propuesta aquí es, incluir en los cálculos previos, el ingrediente modular tipo LEGO y formular varios modelos para ver tendencias de comportamiento, principalmente emitancia y valores preliminares de apertura dinámica.

En la Fig. 15 se muestran, mediante líneas de colores, varias tendencias en una gráfica de emitancia contra cir-

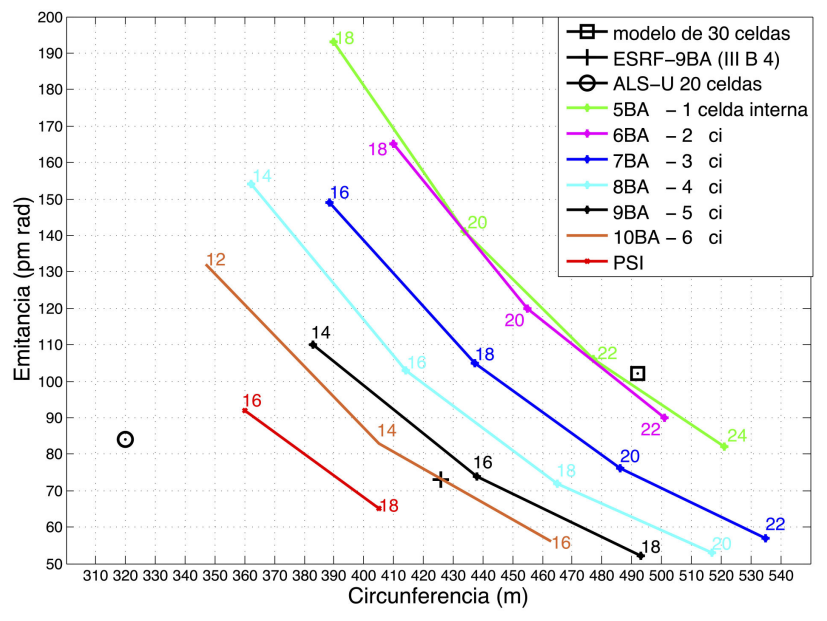

FIGURA 15. En la gráfica se muestran varios modelos en el esquema ESRF+SLS. De ESRF-EBS se toman principalmene los extremos de las celdas que tienen la prominencia de dispersión y se acopla a celdas interiores de SLS-2 en un esquema de estructuras fijas tipo LEGO, desde 1 celda interna (ci) en color verde hasta 6 celdas internas en color naranja. Esto permite relacionar los parámetros de emitancia y circunferencia a $3 \mathrm{GeV}$, dependiendo del número de celdas internas de SLS-2 que se incluyen. Por ejemplo, la línea de color azul representa anillos de 16, 18, 20 y 22 celdas con 3 celdas internas y su estructura es tipo 7BA, como ESRF-EBS, pero con celdas internas de SLS-2, mientras que la línea de color naranja representa el comportamiento de anillos con celdas tipo 10BA con 12, 14 y 16 celdas. En color rojo se muestran modelos de 16 y 18 celdas sugeridos por personal de PSI. Se incluye también como referencia modelos de sincrotrones estudiados en secciones anteriores de este trabajo, ALS-II en (o), ESRF-EBS en (+) y un diseño no incluído aquí, derivado de SLS-2 mediante la idea de módulos tipo LEGO de 30 celdas ( $\square$ ).

cunferencia del sincrotrón. El marco principal que se desea mostrar es el arreglo por estructuras tipo LEGO, cuando se varía el número de celdas internas. Es importante señalar que estas celdas interiores son únicamente celdas de SLS-2 [16]. Cada línea de color se asocia a un número fijo de celdas internas que se han acoplado al segmento, tipo ESRF-EBS, que contiene la prominencia de dispersión entre dos LGB's. Este segmento se ha adaptado a las celdas internas de SLS-2, por lo que permanece sin cambio a lo largo del análisis, es decir, la distribución e intensidad magnética de sus multipolos permanecen fijas para los diferentes colores de las curvas.

El conjunto que presenta mayor emitancia está dado en color verde, que sólo contiene una celda interna de SLS-2. Los números sobre los puntos de la línea indican modelos de AA de 18, 20, 22 y 24 celdas con una celda interior.

En color magenta se muestran tres puntos correspondientes a AA de 18, 20 y 22 celdas con 2 celdas internas.

La línea de color azul representa AA de 16, 18, 20 y 22 celdas con 3 celdas internas. De hecho este conjunto es 7BA, como ESRF-EBS, pero el interior de la celda contiene celdas internas de SLS-2. El AA de 16 celdas es similar al modelo tratado en la Sec. 3.2.1, pero su emitancia y circunferencia son menores. 


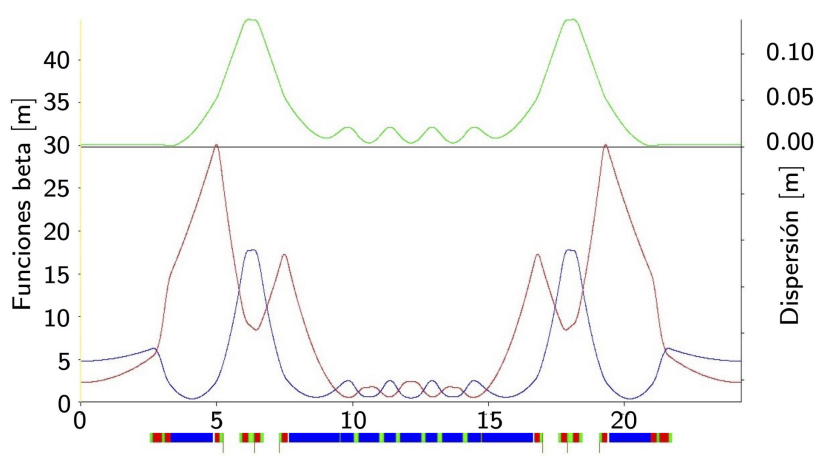

FIGURA 16. Esta figura representa el modelo de 20 celdas de baja emitancia mostrado en la Fig. 15 en color azul. Los extremos de la celda son tipo ESRF-EBS con prominencia de dispersión, mientras que el interior de la celda contiene tres celdas internas de SLS-2. El anillo se cierra con 20 de estas celdas dando una cicunferencia de $486 \mathrm{~m}$ y una emitancia de $77 \mathrm{pm} \cdot \mathrm{rad}$.

TABLA IX. Parámetros principales del modelo considerado en la Fig. 16.

\begin{tabular}{cc}
\hline Parámetro & Valor \\
\hline Energía & $3 \mathrm{GeV}$ \\
Circunferencia & $486 \mathrm{~m}$ \\
Número de celdas & 20 \\
Tono betatrón $\left(\nu_{x}, \nu_{y}\right)$ & $49.799,27.031$ \\
Cromaticidad natural $\left(\xi_{x}, \xi_{y}\right)$ & $-95.2,-80.58$ \\
Emitancia $\left(\epsilon_{x}\right)$ & $77 \mathrm{pm} \cdot \mathrm{rad}$ \\
Factor de compactación de momento $\left(\alpha_{c}\right)$ & $1.2 \times 10^{-4}$ \\
\hline
\end{tabular}

La línea de color cyan representa el comportamiento de AA de 14, 16, 18 y 20 celdas con 4 celdas internas.

La línea de color negro representa el comportamiento de AA de 14,16 y 18 celdas con 5 celdas internas. Este esquema corresponde a celdas tipo 9BA y por tanto podremos compararlo al modelo tratado en la Sec. 3.2.4 de ESRF-ESB, con características $9 \mathrm{BA}+\mathrm{AB}$, que aparece en la gráfica con el símbolo (+).

La línea de color naranja representa el comportamiento de AA de 12, 14 y 16 celdas con 6 celdas internas. Este esquema corresponde a celdas tipo 10BA. Se podría hacer una comparación parcial de este esquema con un diseño 10BA para un sincrotrón de 12 celdas, 324 m, $2.4 \mathrm{GeV}$ y $\sim 120$ $\mathrm{pm} \cdot \operatorname{rad}[21]$.

En la misma gráfica, como referencia, se muestran también otros modelos de sincrotrones que se han mencionado anteriormente, especialmente los de baja emitancia, como los de las secciones ALS-II (3.1) mostrado en (o), los propuestos por miembros de PSI (Fig. 14 y línea roja de la Fig. 15) y un diseño no incluído aquí, derivado de SLS-2 mediante la idea de módulos tipo LEGO de 30 celdas ( $\square$ ).

Para concretar uno de estos diseños en una imagen, tomamos el segundo punto más bajo de la línea azul de la Fig. 15 que representa un diseño de circunferencia menor de $500 \mathrm{~m}$

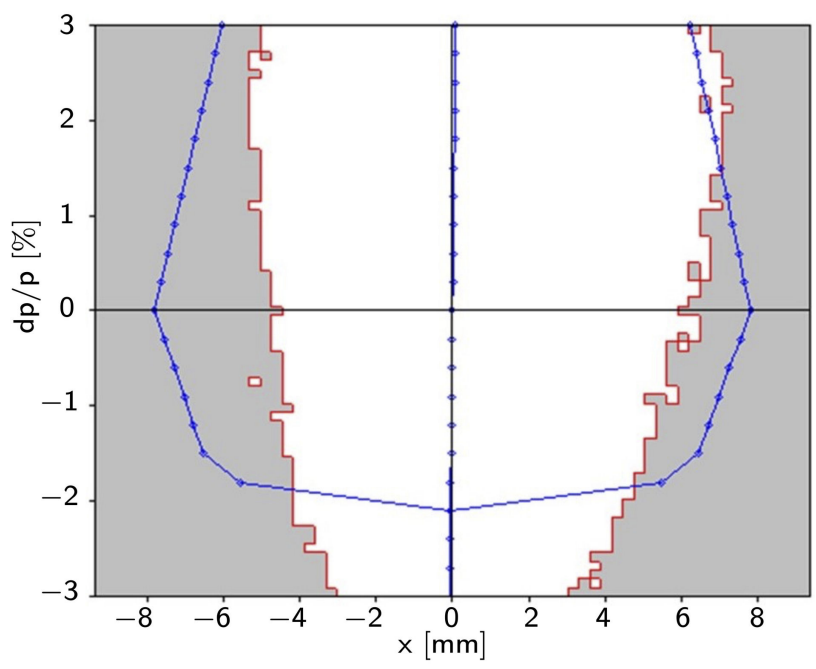

FIGURA 17. Apertura de momento al inicio de la celda para el diseño tipo ESRF + SLS-2 de 7BA de la Fig. 16 de $77 \mathrm{pm} \cdot \mathrm{rad}$ Aparentemente es susceptible de mejorarse con un método de optimización más robusto.

(486) con emitancia baja (77 pm · rad). Otros parámetros del modelo se muestran en la Tabla IX. En la Fig. 16 se muestra la distribución magnética combinada de ESRF-ESB y SLS-2 así como las funciones ópticas asociadas al modelo. Este modelo tiene también una apertura dinámica heredada que se ve atractiva para mejorarse como se muestra en la Fig. 17.

\section{Discusión y conclusiones}

Se han presentado varias estructura magnéticas basadas en modelos recientes de actualizaciones de fuentes de luz como ALS-II, ESRF-EBS y SLS-2, así como un estudio modular tipo LEGO de una combinación ESRF-EBS/SLS-2 que permite seguir el comportamiento de algunos de sus parámetros, en especial una emitancia baja, el tamaño del sincrotrón y el número factible de líneas experimentales.

Un punto de vista crítico de los resultados de la Fig. 15 muestra que el diseño basado en ALS-II puede tener buen desempeño, es de baja emitancia, tamaño reducido, 20 secciones rectas, pero dada su baja apertura dinámica (en nuestro caso no completamente optimizada) requiere adicionalmente de un anillo acumulador para el proceso de inyección sobre el eje.

El diseño tipo ESRF-EBS, 9BA, 16 celdas con $\mathrm{AB}$ realizado en forma independiente al esquema LEGO es competitivo en sus parámetros con el correspondiente de 16 celdas en el formato tipo LEGO. La aperturas dinámicas (con optimización ligera) parecen ser mayores de 4-5 $\mathrm{mm}$ en ambos casos.

Los resultados del punto 12 naranja (12 celdas en el anillo, con 6 celdas interiores de SLS-2, entre regiones de ESRFEBS con alta dispersión), parecen ser también consistentes con otros modelos 10 BA, como el de la Ref. [24] realizados a $2.4 \mathrm{GeV}$, con apertura dinámica mayor de $10 \mathrm{~mm} \sin$ cavidades de radiofrecuencia y errores. 
El modelo estudiado en forma independiente al esquema tipo LEGO $(\square)$, resalta por las 30 celdas que contiene, con emitancia y circunferencia aceptables. Cálculos preliminares de su apertura dinámica sugieren $4 \mathrm{~mm}$.

El esquema introducido tipo LEGO, en modelos ESRFEBS+SLS-2, permite visualizar en general varios modelos magnéticos con sus correspondientes parámetros de emitancia, tamaño del anillo y secciones rectas. Sus aperturas dinámicas pueden variar y será necesario modificar los avances de fase por celda, horizontal y vertical, para lograr mejorar la apertura dinámica de una distribución magnética específica, de las mostradas en la Fig. 15, que sea de interés particular.

\section{Agradecimientos}

Nuestro profundo agradecimiento para Rafael Abela por su interés en el trabajo, Albin Wrulich por discusiones que se reflejan en el punto 3.3.3 y Andreas Streun por compartir amablemente la versión v.b025 de SLS-2. AA agradece a Joan Bordas sus valiosas discusiones.

Investigación realizada gracias al programa UNAMPAPIIT IN108522.
1. R. P. Walker, Potentialities and compromises in the design of diffraction limited storage rings, International ICFA miniWorkshop on Nonlinear dynamics and Collective Effects in particle beam physics, September (2017).

2. D. Einfeld and M. Plesko, A modified QBA optics for low emittance storage rings, Nuclear Instruments and Methods in Physics Research Section A: Accelerators, Spectrometers, Detectors and Associated Equipment 335 (1993) 402, https: //doi.org/10.1016/0168-9002(93)91224-B

3. D. Einfeld and M. Plesko, Design of a diffraction-limited light source. Proc. SPIE, Electron-beam sources of High Brightness Radiation, (1993).

4. D. Einfeld, M. Plesko, and J. Schaper. First multi-bend achromat lattice consideration. Jnl of Synchrotron Radiation, 21 (2014) 856. https://doi.org/10.1107/ S160057751401193X

5. R. Bartolini, R. Hettel, and F. Pérez. Comunicación privada. III Mexican Workshop on Accelerator Physics, Huatulco, (2014).

6. C. Steier, Possibilities for a diffraction-limited upgrade of a soft X-ray light source. Synchrotron Radiation News, 27 (2014) 18, htts://doi.org/10.1080/08940886. 2014.970934

7. A. Streun, Opa, lattice design code. htts://ados.web. psi.ch/opa/ (2012).

8. H. Tarawneh et al, ALS-II, a Potential Soft X-ray, Diffraction Limited Upgrade of the Advanced Light Source, J. Phys.: Conference Series 17th Pan-American Synchrotron Radiation Instrumentation Conference 493 (2014) 012020, $10.1088 /$ 1742-6596/493/1/012020

9. C. Steier et al., Proposal for a Soft X-ray Diffraction Limited Upgrade of the ALS, IPAC14, (2014). http://als.lbl.gov/als_physics/csteier/ talks/alsu_poster_ipac14.pdf

10. L. Farvacque et al., A low-emittance lattice for the esrf. IPAC'13, Proc. IPAC2013 MOPE008 (2013) 79, https://accelconf.web.cern.ch/IPAC2013/ papers/mopea008.pdf

11. R. Dimper, H. Reichert, P. Raimondi, L. Sánchez Ortiz, F. Sette, and J. Susini, Esrf upgrade programme phase ii (2015-2022). page 7, (2014), htts://www.esrf.fr/Apache_files/ Upgrade/ESRF-orange-book.pdf

12. Y. Jiao Xu, and Y.-M. Peng, ESRF-type lattice design and optimization for the high energy photon source, Chinese Physics C 40 (2016) 027001, https://doi.org/10.1088/ 1674-1137/40/2/027001.

13. R. Hettel (APS-U Project Director). The advanced photon source upgrade plan approved. Synchrotron Radiation News, 32 (2019) 34, htts://doi.org/10.1080/08940886. 2019.1582289 .

14. C. Abraham et al., Diamond-ii conceptual design report. (2019). htts://www.diamond.ac.uk/dam/jcr: ec67b7e1-fb91-4a65-b1ce-f646490b564d/ Diamond-II $\% 20$ Conceptual\%20Design\% 20Report.pdf

15. L.O. Dallin. Design considerations for an ultralow emittance storage ring for the canadian light source. 9th International Particle Accelerator Conference IPAC2018 TUPMF038 (2018) 1334, https://doi.org/10. $18429 /$ JACOWIPAC2018-TUPMF 038

16. M. Borland, ELEGANT: A exible SDDS-compliant code for accelerator simulation, Advanced Photon Source LS-287, (2000) 1, https://doi.org/10.2172/761286

17. K. Soutome and H. Tanaka, Higher-order formulas of amplitude-dependent tune shift caused by a sextupole magnetic field distribution, Phys. Rev. Accel. Beams, 20 (2017) 064001, https://doi.org/10.1103/ PhysRevAccelBeams.20.064001

18. L. O. Dallin, Cls 2.2: Ultra-Brilliant Round Beams Using Pseudo Longitudinal Gradient Bends. In 10th Int. Particle Accelerator Conf. TUPGW004 (2019) 1385, https://doi.org/ $10.18429 /$ JACOW-IPAC2019-TUPGW004

19. A. Streun, Comunicación privada. la versión v.b025 ha sido amablemente compartida por A. streun. (2019).

20. A. Wrulich, Comunicación privada. (2019).

21. D. Einfeld, J. Schaper, and M. Plesko, Design of a diffraction limited light source (di). Proceedings Particle Accelerator Conference, IEEE, 1 (1995) 177. https://doi.org/10.1109/ PAC.1995.504602. 
22. R. Nagaoka and A. F. Wrulich, Emittance minimisation with longitudinal dipole field variation. Nucl. Instrum. Methods Phys. Res. A, 575 (2007) 292. https://doi.org/10. $1016 / j . n i m a .2007 .02 .086$

23. A. Streun, The anti-bend cell for ultralow emittance storage ring lattices. Nucl. Instrum. Methods Phys. Res. A,
737 (2014) 148,https://doi.org/10.1016/j.nima. 2013.11 .064

24. P. Yang, Z. Bai, T. Zhang, D. Xu, and L. Wang, Design of a hybrid ten-bend-achromat lattice for a diffraction-limited storage ring light source. Nucl. Instrum. Methods Phys. Res. A 943 (2019) 162506. https://doi.org/10.1016/j.nima. 2019.162506 